\title{
A CASE OF CAPLAN'S SYNDROME IN A BOILER-SCALER
}

\author{
BY \\ J. A. CAMPBELL \\ From the Department of Pathology, Broadgreen Hospital, Liverpool
}

(RECEIVED FoR PUblication JANUARY 8, 1958)

In 1953 Caplan recorded a high incidence of massive shadows in the chest radiographs of coalminers with rheumatoid arthritis, and described a particular type of radiological opacity occurring in about $25 \%$ of these arthritics. The shadows differed from the usual form of massive fibrosis in pneumoconiosis, in that they were numerous, round, well-defined, and scattered evenly throughout both lung fields. They were more rapid in their development than in progressive massive fibrosis, reaching a diameter of 1 to $2 \mathrm{~cm}$. and occasionally as much as $5 \mathrm{~cm}$. in a few months, thereafter remaining stationary. Where the rounded opacities appeared, the background of simple pneumoconiosis was usually slight, and where a more severe form of pneumoconiosis was present the radiological appearances were usually those of progressive massive fibrosis.

The only account of the pathology of Caplan's syndrome that $I$ have been able to find is that of Gough, Rivers, and Seal (1955). All the patients described were coal-miners. Gough does, however, quote a paper by Colinet describing a similar radiological picture in silica workers, but without pathological studies.

In view of the paucity of descriptions of the syndrome in conditions other than in coal-miner's lung, the following case of Caplan's syndrome in a ship's boiler-scaler is reported.

\section{Case Report}

The patient, aged 62 years at his death, was employed as a ship's boiler-scaler for the greater part of his life. He took up this occupation at the age of 13 years, continuing the same trade for 31 years until 1939, when he gave it up because of his "chestiness." He then became an electrician's mate, an occupation which he followed until rheumatoid arthritis made him give that up in 1951 .

The rheumatoid arthritis began in May, 1951, involving both shoulders, the neck, the right knee, and both feet. He was treated at a hospital out-patient department with gold injections, codeine tablets, and physiotherapy. A chest radiograph was taken at the time and showed pneumoconiosis.
In November, 1952, he was admitted to hospital with severe chronic bronchitis and rheumatoid arthritis. At that time he was cyanosed and dyspnoeic and had a severe cough with copious black sputum. The same joints as before were affected by rheumatoid arthritis, and he was treated with gold injections.

A radiograph of the chest taken at this time was reported as showing "very fine reticulation throughout, consistent with boiler-scaler's lungs, but pulmonary tuberculosis cannot be excluded as there are nodular lesions with cavitation in both upper zones typical of tuberculosis." Comparison with a radiograph taken a year previously at the Out-patient Clinic showed no change except possible cavitation in the left upper zone. During this stay in hospital the sputum was examined eight times for tubercle bacilli and cultured once, all with negative results.

In July, 1954, he was admitted to Broadgreen Hospital, under the care of Mr. Heron, with a severe flare-up of the rheumatoid arthritis which now involved the neck, both shoulders, elbows, and

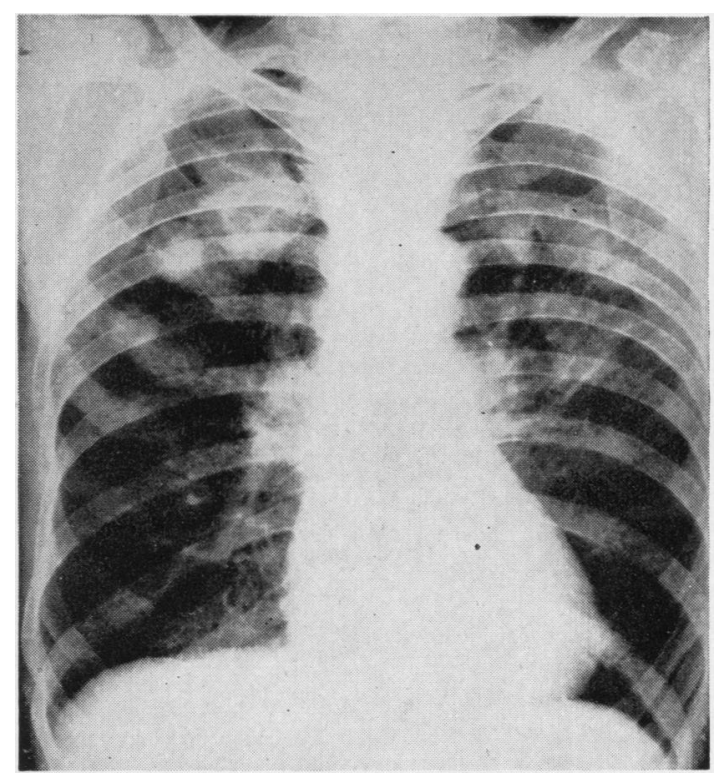

FIG. 1.-Radiograph taken in 1954 showing the characteristic "rheumatoid" nodules in the right upper lobe. 


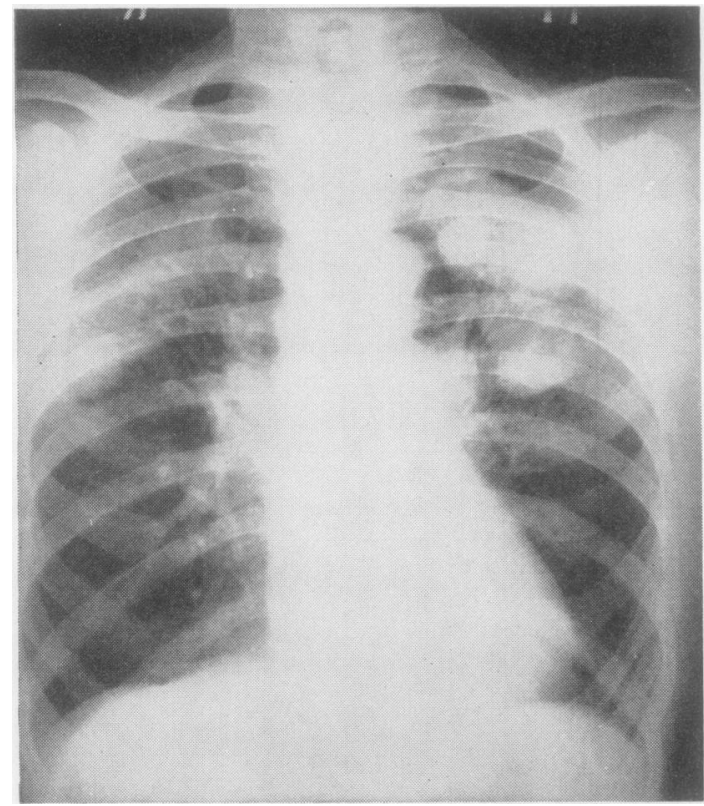

FIG. 2.-Radiograph taken in 1956 showing extension of the disease with opacities in the left upper lobe.

wrists, and to a less extent the knee and ankle joints. There was a marked deformity of both feet due to subluxation dorsally of all the toes, which was later treated by amputation of the toes, followed after an interval of one year by an operation for mid-tarsal fusion. At the time of admission in 1954 he was cyanosed but showed no evidence of finger clubbing. A radiograph of the chest was reported by Dr. Rubin as showing "a diffuse silicotic nodulation of both lungs, with multiple round foci in both lungs very suggestive of silicotic lesions in a subject suffering from rheumatoid arthritis" (Fig. 1). Unfortunately the films taken in 1951 and 1952 were not available for comparison. The E.S.R. at this time was $39 \mathrm{~mm}$. (Wintrobe). The patient was given a prolonged course of physiotherapy and by March, 1956, was well enough to consider retraining for a job.

Five months later, however, he was readmitted with a severe flare-up of arthritis in all joints, especially the shoulders, for which he was treated with cortisone, corticotrophin, and aspiration of both shoulder joints. A chest radiograph taken at this time showed an increase in the nodular shadowing with extensive pulmonary fibrosis. The appearance was more like massive fibrosis (Fig. 2).

In October, 1956, his cough became worse and he had copious black sputum. $\mathrm{He}$ soon became asthmatic and eventually required an oxygen tent. Sputum examinations and cultures for tubercle bacilli were negative on two occasions. By December he was in congestive heart failure and he died at the end of January, 1957.

\section{NECROPSY}

Macroscopic Appearances.-The necropsy was performed by Dr. Whitwell. There was oedema of both lower limbs. The joints of both hands and feet showed severe deformities which were typical of rheumatoid arthritis. There were anterior subluxations of both hands at the wrist joints, and all the toes had been amputated. The knees and the right shoulder were swollen.

Pleural adhesions were present on both sides. The lungs were bulky, nodular, and heavily pigmented, and when they were sectioned a large amount of black fluid flowed from the cut surface. There was some bullous emphysema at the apices and along the margins of both lungs. The cut surfaces showed multiple small pigmented fibrous nodules, measuring about 1 to $5 \mathrm{~mm}$. in diameter. There was focal

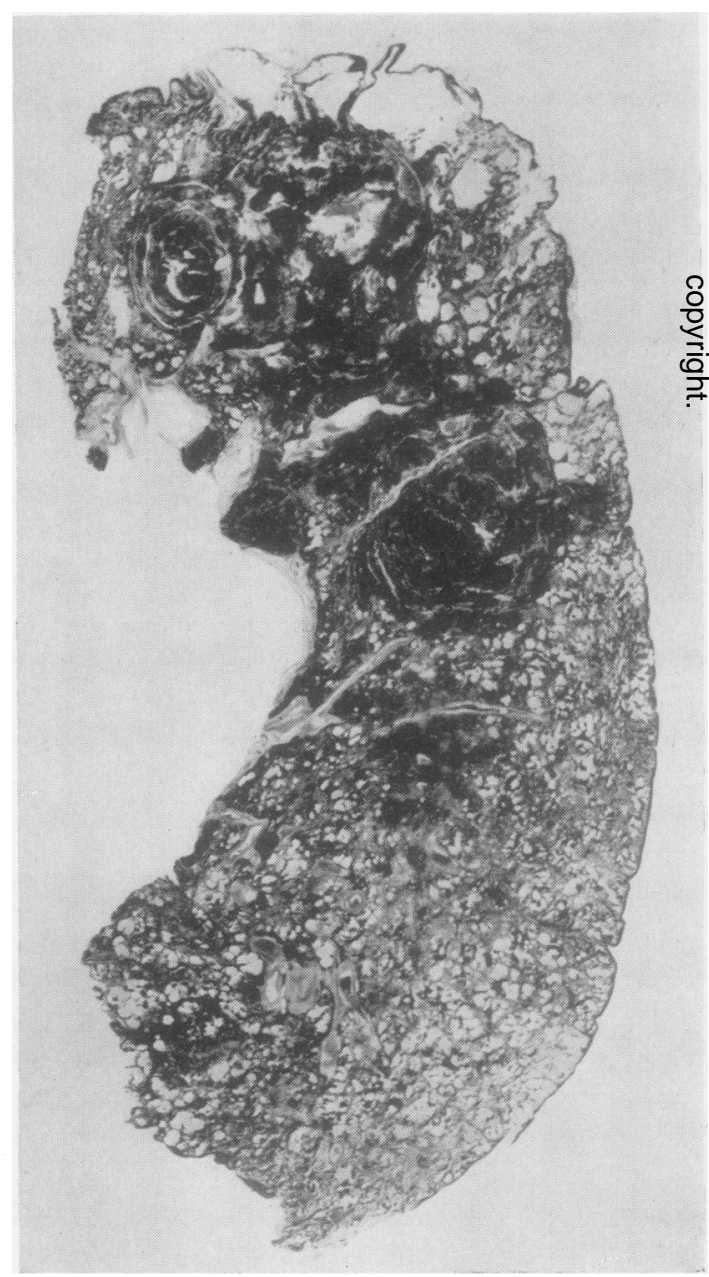

FIG. 3.-Unstained section of the left upper lobe, showing the concentric markings in the nodules. 
emphysema around these lesions. In addition there were larger circumscribed nodules in both lungs mainly in the apices of the upper lobes. The largest of these measured $4 \mathrm{~cm}$. in its greatest diameter. They were black and appeared to be made up of several concentric black layers with intervening yellow or grey tissue. Two of the larger nodules in the apex of the left upper lobe were confluent, but otherwise they were discrete (Fig. 3). There was no evidence of tuberculosis in either lung. The base of the left lower lobe contained several infarcts. The hilar lymph nodes showed extensive pigmentation with moderate fibrosis.

The heart was enlarged, and the right ventricle was dilated and hypertrophic. The right atrium and the great veins were distended with blood. There was some atheroma of the pulmonary arteries. The liver showed severe chronic congestion, and the other organs although normal were also congested.

Microscopic APPEARANCES.-There were dust cells in some of the alveoli, but they were not numerous.
The smaller nodules were irregular in shape and deeply pigmented. They consisted mainly of reticulin, but some of them contained collagen, which was arranged irregularly or in a radial fashion, but never concentrically. There was emphysema around the pigmented nodules, which were largely perivascular. There was also a considerable amount of pigment in the subpleural lymphatics and around the bronchioles. None of the pigment gave the staining reactions of iron.

Examination of the large nodules showed that they had collagenous centres, with varying degrees of necrosis. A considerable amount of dust was present (Fig. 4), usually arranged irregularly but with a tendency to form concentric rings. The necrotic centre was surrounded by dense collagen arranged circumferentially, inside which was a zone of inflammation consisting of polymorphs and macrophages, with some nuclear remnants. This zone was often separated from the surrounding collagen by a cleft. The outer collagen was infiltrated with lymphocytes,

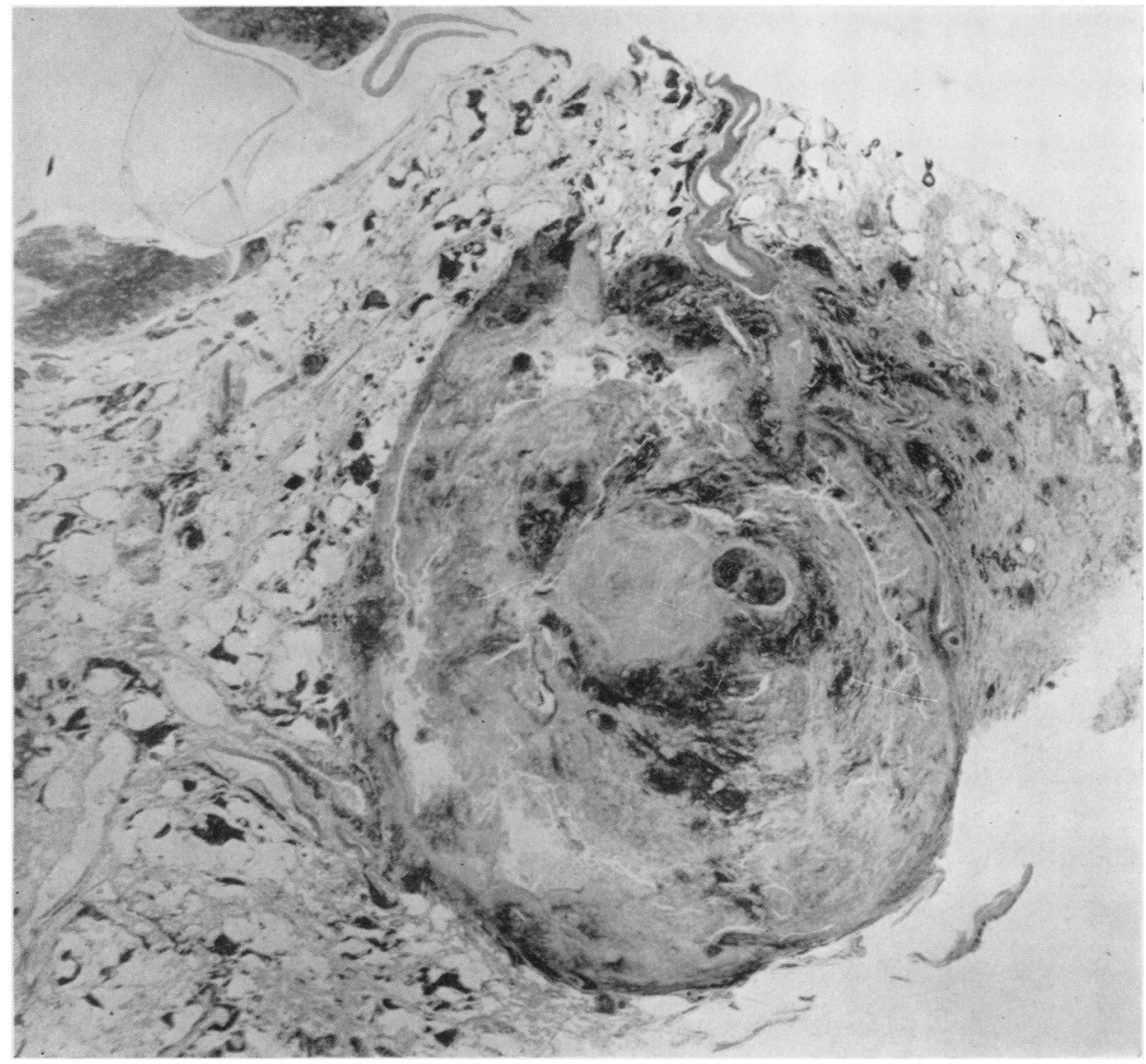

FIG. 4.-A " rheumatoid" nodule showing deposition of dust in a roughly concentric manner. It is surrounded by simple coal nodules with focal emphysema. Haematoxylin and eosin. $\times 4$. 
plasma cells, and occasional polymorphs. The central collagen fibres ended abruptly at the edge of the inflammatory zone. Some of the foci showed areas of calcification. Periodic-acid-Schiff staining of the nodules was faint.

\section{COMMENT}

The naked-eye and histological appearance of the lungs of boiler-scalers with pneumoconiosis is identical with that seen in the pneumoconiosis of South Wales coal-miners (Harding and Massie, 1951) and with the general appearance of the lungs in this patient. The superimposed changes seen in this patient, including the non-specific inflammatory zone which may be the " rheumatoid" component, are the same as those described by Gough, Rivers, and Seal (1955). These authors found evidence of tuberculosis in only five out of their 16 cases, which is about the same incidence as is found in progressive massive fibrosis. In the present case, apart from a suggestion of tuberculous cavitation in the 1952 radiograph, there has never been any evidence of tuberculosis either in the sputum on direct examination or culture, or on histological examination of the necropsy material.

My thanks are due to Dr. Whitwell for permission to publish this case, and to Professor Gough for the large paper section of lung and also for the photographs of radiographs.

\section{REFERENCES}

Caplan, A. (1953). Thorax, 8, 29.

Gough, J., Rivers, D., and Seal, R. M. E. (1955). Ibid., 10, 9

Harding, H. E., and Massie, A. P. (1951). Brit.J. industr. Med., 8, 256. 